\title{
Correction to: Magnetic Resonance Imaging of Deltoid Muscle/Tendon Tears: A Descriptive Study
}

\section{${\text { Ceylan } \text { Colak }^{1} \text { (D) Jennifer A. Bullen }}^{2} \cdot$ Vahid Entezari $^{3} \cdot$ Michael Forney $^{1} \cdot$ Hakan Ilaslan $^{1}$}

Published online: 3 April 2021

(C) ISS 2021

\section{Correction to: Skeletal Radiology}

https://doi.org/10.1007/s00256-021-03727-6

The name of Vahid Entezari was incorrectly captured in the original manuscript.

The correct authorname should be:

$<$ GivenName $>$ Vahid </GivenName $>$

$<$ FamilyName $>$ Entezari</FamilyName $>$

The original article has been corrected.

Publisher's note Springer Nature remains neutral with regard to jurisdictional claims in published maps and institutional affiliations.

The online version of the original article can be found at https://doi.org/ $10.1007 / \mathrm{s} 00256-021-03727-6$

\section{Ceylan Colak}

colakc@ccf.org

1 Imaging Institute, Cleveland Clinic, 9500 Euclid Ave, Cleveland, OH 44195, USA

2 Quantitative Health Sciences, Cleveland Clinic, Cleveland, $\mathrm{OH}$, USA

3 Department of Orthopaedic Surgery, Cleveland Clinic, Cleveland, OH, USA 